\title{
Neurofilament ELISA validation
}

September 28, 2009 


\section{Authors:}

1. Petzold, Axel ${ }^{1}$

2. Altintas, Ayse $^{2}$

3. Andreoni, Laura ${ }^{3}$

4. Bartos, Ales ${ }^{4}$

5. Berthele, Achim ${ }^{5}$

6. Blankenstein, M.A. ${ }^{6}$

7. Buee, $\mathrm{Luc}^{7}$

8. Castellazzi, Massimiliano ${ }^{8}$

9. Cepok, Sabine ${ }^{9}$

10. Comabella, Manuel ${ }^{10}$

${ }^{1}$ Department of Neuroinflammation, UCL Institute of Neurology, Queen Square, London WC1N 3BG, United Kingdom. Tel. +44 (0)20 78373611 ext: 4204, Fax +44 (0)20 7837 8553. Email: a.petzold@ion.ucl.ac.uk

${ }^{2}$ Istanbul University, Cerrahpasa Medical Faculty, Department of Neurology, Istanbul, Turkey. Email: altintas13@gmail.com

${ }^{3}$ Lab. of Neuroimmunology, IRCCS, Neurological Institute 'C. Mondino', I-27100 Pavia, Italy Email: laura.andreoni@mondino.it

${ }^{4}$ AD Center, Prague Psychiatric Center, Prague, Czech Republic and Charles University in Prague, Third Faculty of Medicine, University Hospital Kralovske Vinohrady, Department of Neurology, Prague, Czech Republic. Email: bartos@pcp.If3.cuni.cz

${ }^{5}$ Department of Neurology, Klinikum rechts der Isar, Technische Universität Ismaninger Strae 2281675 München, Germany. Email: berthele@|rz.tu-muenchen.de

${ }^{6}$ Lab. Klinische Chemie PK 1 BR 011, VU Medisch Centrum, De Boelelaan 1117, Amsterdam, The Netherlands. Email: MA.Blankenstein@vumc.nl

${ }^{7}$ Inserm U837 - JPArc Alzheimer \& Tauopathies Université Lille 2 Faculté de MédecinePôle Recherche Institut de Médecine Prédictive et Recherche Thérapeutique 59045 Lille France. Email: luc.buee@inserm.fr

${ }^{8}$ Laboratorio di Neurochimica, Sezione di Clinica Neurologica, Dipartimento di Discipline Medico Chirurgiche della Comunicazione e del Comportamento Università degli Studi di Ferrara, Ferrara, Italy. Email: massimiliano.castellazzi@unife.it

${ }^{9}$ Department of Neurology, Klinikum rechts der Isar, Technische Universität Ismaninger Strae 2281675 München, Germany. Email: cepok@Irz.tu-muenchen.de

${ }^{10}$ Department of Neurology, Centre d'Esclerosi Múltiple de Catalunya, Unitat de Neuroimmunologia Clínica, Hospital Universitari Vall d'Hebron,Barcelona, Spain. Barcelona, Spain. Email: mcomabel@ir.vhebron.net 


\section{Constantinescu, Cris $S^{11}$}

\section{Deisenhammer, Florian ${ }^{12}$}

13. Deniz, Gunnur ${ }^{13}$

\section{Erten, Gaye ${ }^{14}$}

15. Espiño, Mercedes ${ }^{15}$

\section{Fainardi, Enrico ${ }^{16}$}

\section{Franciotta, Diego ${ }^{17}$}

18. Freedman, Mark ${ }^{18}$

19. Giedraitis, Vilmantas ${ }^{19}$

20. Gilhus, Nils Erik ${ }^{20}$

\section{Giovannoni, Gavin ${ }^{21}$}

\section{Glabinski, Andrzejej}

\footnotetext{
${ }^{11}$ Division of Clinical Neurology University of Nottingham, Medical School, Nottingham, UK. Email: Cris.Constantinescu@nottingham.ac.uk

${ }^{12}$ Clinical Dept. of Neurology, Innsbruck Medical University, Anichstrasse 35, A-6020 Innsbruck. Email: florian.deisenhammer@i-med.ac.at

${ }^{13}$ Istanbul University, Institute of Experimental Medicine, Istanbul, Turkey. Email: gdeniz@istanbul.edu.tr

${ }^{14}$ Istanbul University, Institute for Experimental Medicine, Department of Immunology, Istanbul, Turkey. Email: gerten@istanbul.edu.tr

${ }^{15}$ Immunology Department. Hospital Ramon y Cajal, Madrid, Spain. Email: mespino.hrc@salud.madrid.org

${ }^{16}$ Neuroradiology Unit, Azienda Ospedaliero-Universitaria, Ferrara, Italy. Email: e.fainardi@ospfe.it

${ }^{17}$ Lab. of Neuroimmunology, IRCCS, Neurological Institute 'C. Mondino', I-27100 Pavia, Italy. Email: diego.franciotta@mondino.it

${ }^{18}$ Ottawa Health Research Institute. Ottawa Hospital-General Campus, Ottawa, Ontario, Canada. Email: MFREEDMAN@ottawahospital.on.ca

${ }^{19}$ Uppsala University, Department of Public Health/Geriatrics, Rudbeck Laboratory, Uppsala Science Park, Uppsala, Sweden. Email: Vilmantas.Giedraitis@pubcare.uu.se

${ }^{20}$ Department of Clinical Medicine, University of Bergen; and Department of Clinical Medicine, University of Bergen, and Department of Neurology Haukeland University Hospital, Bergen, Norway. Email: nils.gilhus@helse-bergen.no

${ }^{21}$ Neuroimmunology Unit, ICMS, Barts and The London School of Medicine and Dentistry, London, UK. Email: g.giovannoni@qmul.ac.uk

${ }^{22}$ Department of Propedeutics of Neurology, Medical University of Lodz, Kopernik Hospital, Pabianicka Str. 62, Lodz, Poland. Email: aglabinski@gmail.com
} 
23. Grieb, Pawel ${ }^{23}$

24. Hartung, Hans-Peter ${ }^{24}$

25. Hemmer, Bernhard ${ }^{25}$

26. Herukka, Sanna-Kaisa ${ }^{26}$

27. Hintzen, Rogier ${ }^{27}$

28. Ingelsson, Martin ${ }^{28}$

29. Jackson, Samuel ${ }^{29}$

30. Jacobsen, Steve ${ }^{30}$

31. Jafari, Naghmeh ${ }^{31}$

32. Jalosinski, Marcin ${ }^{32}$

33. Jarius, $\operatorname{Sven}^{33}$

34. Kapaki, Elisabeth ${ }^{34}$

\footnotetext{
${ }^{23}$ Medical Research Center, Polish Academy of Sciences, 02-106 Warsaw, Pawinskiego 5; Poland. Email: pgrieb@cmdik.pan.pl

${ }^{24}$ Department of Neurology, Heinrich-Heine University, Dsseldorf, Germany. Email: Hans-Peter.Hartung@uni-duesseldorf.de

${ }^{25}$ Department of Neurology, Klinikum rechts der Isar, Technische Universität Ismaninger Strae 2281675 München, Germany. Email: hemmer@Irz.tu-muenchen.de

${ }^{26}$ Department of Neurology University of Kuopio P.O.Box 162770211 Kuopio Finland. Email: Sanna-Kaisa.Herukka@uku.fi

${ }^{27}$ The Department of Neurology, MS Centre ErasMS, Erasmus MC, Rotterdam, The Netherlands. Email: rhintzen@xs4all.nl

${ }^{28}$ Uppsala University, Dept. of Public Health / Geriatrics, Rudbeck Laboratory, Dag Hammarskjölds väg 20, 75185 Uppsala, Sweden. Email: martin.ingelsson@pubcare.uu.se

${ }^{29}$ Neuroimmunology Unit, ICMS, Barts and The London School of Medicine and Dentistry, London, UK Email: s.j.jackson@qmul.ac.uk

${ }^{30} \mathrm{NINDS} / \mathrm{NIH}$, Viral Immunology Section, 9000 Rockville Pike, Building 10. Email: jacobsons@ninds.nih.gov

${ }^{31}$ The Department of Neurology, MS Centre ErasMS, Erasmus MC, Rotterdam, The Netherlands. Email: n.jafari@erasmusmc.nl

${ }^{32}$ Department of Propedeutics of Neurology, Medical University of Lodz, Kopernik Hospital, Pabianicka Str. 62, Lodz, Poland. Email: marcin.jalosinski@gmail.com

${ }^{33}$ Division of Molecular Neuroimmunology, Department of Neurology, University of Heidelberg, Germany. Email: Sven.Jarius@med.uni-heidelberg.de

${ }^{34}$ Department of Neurology, Athens National University, "Eginition" Hospital, 74 Vass. Sofias Ave., Athens 11528, Greece. Email: ekapaki@med.uoa.gr
} 


\section{Kieseier, Bernd $C^{35}$}

36. Koel-Simmelink, Marleen J.A. ${ }^{36}$

\section{Kornhuber, Johannes ${ }^{37}$}

38. Kuhle, Jens ${ }^{38}$

39. Kurzepa, Jacek ${ }^{39}$

40. Lalive, Patrice ${ }^{40}$

41. Lannfelt, Lars ${ }^{41}$

42. Lehmensiek, Vera ${ }^{42}$

43. Lewczuk, Piotr ${ }^{43}$

44. Livrea, Paolo 44

\section{Marnetto, Fabiana ${ }^{45}$}

\footnotetext{
${ }^{35}$ Department of Neurology, Heinrich-Heine University, Dsseldorf, Germany. Email: Bernd.Kieseier@uni-duesseldorf.de

${ }^{36}$ NeuroUnit Biomarkers for Inflammation and Neurodegeneration. Department of Molecular Cell Biology and Immunology; VU University Medical Center; Amsterdam; The Netherlands. Email: MJA.Koel-Simmelink@vumc.nl

${ }^{37}$ Department of Psychiatry and Psychotherapy, Universittsklinikum Erlangen, Germany. Email: Johannes.Kornhuber@uk-erlangen.de

${ }^{38}$ Neurology and Clinical Neuroimmunology, University Hospital, University of Basel, Switzerland. Email: jkuhle@uhbs.ch

${ }^{39}$ Departament of Neurology, Medical University of Lublin, 20-954 Lublin, Jaczewskiego 8, Poland. Email: kurzepa@onet.pl

${ }^{40}$ Department of Neurosciences, Division of Neurology, Neuroimmunology Laboratory, and Department of Genetics and Laboratory Medicine, Laboratory Medicine Service; University Hospital of Geneva and Faculty of Medicine, Geneva, Switzerland. Email: Patrice.Lalive@hcuge.ch

${ }^{41}$ Uppsala University, Dept. of Public Health / Geriatrics, Rudbeck Laboratory, Dag Hammarskjölds väg 20, 75185 Uppsala, Sweden. Email: lars.lannfelt@pubcare.uu.se

${ }^{42}$ Department of Neurology, University of Ulm, 89081, Ulm, Germany. Email: vera.lehmensiek@uni-ulm.de

${ }^{43}$ Department of Psychiatry and Psychotherapy, Universittsklinikum Erlangen, Germany. Email: Piotr.Lewczuk@uk-erlangen.de

${ }^{44}$ Dept. of Neurological and Psychiatric Sciences Policlinico-Piazza G. Cesare,11 70100 University of Bari-Italy. Email: plivrea@neurol.uniba.it

${ }^{45}$ Multiple Sclerosis Center ASO San Luigi Gonzaga Orbassano (Turin), Italy. Email:fabiana.marnetto@unito.it
} 
46. Martino, Davide ${ }^{46}$

47. Menge, $\mathrm{Til}^{47}$

48. Norgren, Niklas ${ }^{48}$

49. Papuc, Eva ${ }^{49}$

50. Paraskevas, George $P^{50}$

51. Pirttilä, Tuula ${ }^{51}$

52. Rajda, Cecília ${ }^{52}$

53. Rejdak, Konrad ${ }^{53}$

54. Ricny, Jan ${ }^{54}$

55. Ripova, Daniela ${ }^{55}$

56. Rosengren, Lars ${ }^{56}$

\section{Ruggieri, Maddalena ${ }^{57}$}

${ }^{46}$ Dept. of Neurological and Psychiatric Sciences Policlinico-Piazza G. Cesare,11 70100 University of Bari-Italy. Email: davidemartino@virgilio.it

${ }^{47}$ Department of Neurology, Heinrich-Heine University, Dsseldorf, Germany. Email: menge@uni-duesseldorf.de

${ }^{48}$ UmanDiagnostics, Umea Biotech Incubator, Tvistevägen 48, SE-90736 Umea, Sweden. Email: niklas.norgren@umandiagnostics.se

${ }^{49}$ Departament of Neurology, Medical University of Lublin, 20-954 Lublin, Jaczewskiego 8, Poland. Email: ewapap@yahoo.pl

${ }^{50}$ Department of Neurology, Athens National University, "Eginition" Hospital, 74 Vass. Sofias Ave., Athens 11528, Greece. Email: geoprskvs44@gmail.com

${ }^{51}$ Department of Neurology University of Kuopio P.O.Box 162770211 Kuopio Finland. Email: tuula.pirttila@uku.fi

${ }^{52}$ Department of Neurology, University of Szeged, Semmelweis u.6., H-6725 Szeged, Hungary. Email: cecil@nepsy.szote.u-szeged.hu

${ }^{53}$ Departament of Neurology, Medical University of Lublin, 20-954 Lublin, Jaczewskiego 8, Poland. Departament of Neurology, Medical University of Lublin, 20-954 Lublin, Jaczewskiego 8, Poland. Email: krejdak@yahoo.com

${ }^{54}$ AD Center, Prague Psychiatric Center, Prague, Czech Republic. Email: ricny@pcp.If3.cuni.cz

${ }^{55}$ AD Center, Prague Psychiatric Center, Prague, Czech Republic. Email: ripova@pcp.If3.cuni.cz

${ }^{56}$ Department of Neurology, Institute of Clinical Neuroscience, Sahlgrenska University Hospital, University of Göteborg, SE-413 45 Gteborg, Sweden. Email: Lars.Rosengren@neuro.gu.se

${ }^{57}$ Dept. of Neurological and Psychiatric Sciences Policlinico-Piazza G. Cesare,11 70100 University of Bari-Italy. Email: mruggieri@neurol.uniba.it. 
58. Schraen, Susanna ${ }^{58}$

59. Shaw, Gerry 59

60. Sindic, Christian ${ }^{60}$

61. Siva, Aksel ${ }^{61}$

62. Stigbrand, Torgny ${ }^{62}$

63. Stonebridge, Iva ${ }^{63}$

64. Topcular, Baris ${ }^{64}$

65. Trojano, Maria ${ }^{65}$

66. Tumani, Hayrettin ${ }^{66}$

67. Twaalfhoven, Harry A.M. ${ }^{67}$

68. Vécsei, László68

69. Van Pesch, Vincent ${ }^{69}$

\footnotetext{
${ }^{58}$ Inserm U837 - JPArc Alzheimer \& Tauopathies Université Lille 2 Faculté de MédecinePôle Recherche Institut de Médecine Prédictive et Recherche Thérapeutique 59045 Lille France. Email: susanna.schraen@inserm.fr

${ }^{59}$ Department of Neuroscience, McKnight Brain Institute, 100 Newell Drive, Gainesville, Florida 32610. Email: shaw@mbi.ufl.edu

${ }^{60}$ Neurochemistry Unit, NCHM - Avenue Hippocrate,10 (boite 1352), 1200 BXL, Brussels, Belgium. Email: sindic@nchm.ucl.ac.be

${ }^{61}$ Istanbul University, Cerrahpasa Medical Faculty, Department of Neurology, Istanbul, Turkey. Email: asiva@tnn.net

${ }^{62}$ Department of Immunology, University of Umea, SE-90187 Umea, Sweden. Email: torgny.stigbrand@climi.umu.se

${ }^{63}$ Ottawa Health Research Institute. Ottawa Hospital-General Campus, Ottawa, Ontario, Canada. Email: istonebridge@ohri.ca

${ }^{64}$ Bakirkoy Prof.Dr. Mazhar Osman Teaching and Research Hospital for Mental Health and Neurological Disorders, Istanbul, Turkey Email: btopcular@gmail.com

${ }^{65}$ Dept. of Neurological and Psychiatric Sciences Policlinico-Piazza G. Cesare,11 70100 University of Bari-Italy. Email: mtrojano@neurol.uniba.it

${ }^{66}$ Department of Neurology, University of Ulm, 89081, Ulm, Germany. Email: hayrettin.tumani@uni-ulm.de

${ }^{67}$ Lab. Klinische Chemie PK 1 BR 011, VU Medisch Centrum, De Boelelaan 1117, Amsterdam, The Netherlands. Email: HAM.Twaalfhoven@vumc.nl

${ }^{68}$ Department of Neurology, University of Szeged, Semmelweis u.6., H-6725 Szeged, Hungary. Email: vecsei@nepsy.szote.u-szeged.hu

${ }^{69}$ Universit Catholique de Louvain, Neurochemistry Unit (NCHM) avenue Mounier, 5359. 1200 BXL. Email: Vincent.Vanpesch@uclouvain.be
} 
70. Vanderstichele, Hugo $^{70}$

71. Vedeler, Christian ${ }^{71}$

72. Verbeek, Marcel M. ${ }^{72}$

73. Villar, Luisa Maria ${ }^{73}$

74. Weissert, Robbert ${ }^{74}$

75. Wildemann, Brigitte ${ }^{75}$

76. Yang, $\mathrm{Cui}^{76}$

77. Yao, Karen ${ }^{77}$

78. Teunissen, Charlotte ${ }^{78}$

\footnotetext{
${ }^{70}$ Innogenetics NV, BTW BE 0427.550.660, RPR Gent, Technologiepark 6, B-9052 Gent, Belgium. Email: hugovdr@innogenetics.be

${ }^{71}$ Department of Clinical Medicine, University of Bergen, and Department of Neurology Haukeland University Hospital, Bergen, Norway. Email: christian.vedeler@helsebergen.no

${ }^{72}$ Radboud University Nijmegen Medical Centre, Donders Centre for Brain, Cognition and Behaviour, Department of Neurology, Laboratory of Pediatrics and Neurology, Alzheimer Centre Nijmegen, The Netherlands. Email: M.Verbeek@cukz.umcn.nl

${ }^{73}$ Immunology Department. Hospital Ramon y Cajal, Madrid, Spain. Ivillar.hrc@salud.madrid.org

${ }^{74}$ Service de Neurologie/Neuroimmunologie - HUG, Geneve Departement de Pathologie et Immunologie - CMU, Geneve Email: robert.weissert@hcuge.ch

${ }^{75}$ Division of Molecular Neuroimmunology, Department of Neurology, University of Heidelberg, Germany. Email: Brigitte.Wildemann@med.uni-heidelberg.de

${ }^{76}$ Department of Neuroscience, McKnight Brain Institute, 100 Newell Drive, Gainesville, Florida 32610. Email: yangcui@ufl.edu

${ }^{77} \mathrm{NINDS} / \mathrm{NIH}$, Viral Immunology Section, 9000 Rockville Pike, Building 10. Email: YaoK@ninds.nih.gov

${ }^{78}$ NeuroUnit Biomarkers for Inflammation and Neurodegeneration. Department of Molecular Cell Biology and Immunology; VU University Medical Center; Amsterdam; The Netherlands. Email:c.teunissen@vumc.nl
} 


\begin{abstract}
Background: Neurofilament proteins (Nf) are highly specific biomarkers for neuronal death and axonal degeneration. As these markers become more widely used, an inter-laboratory validation study is required to identify assay criteria for high quality performance.

Methods: The UmanDiagnostics NF-light $\AA^{\circledR}$ enzyme-linked immunoabsorbant assays (ELISA) for the neurofilament light chain (NfL, $68 \mathrm{kDa}$ ) was used to test the intra-assay and inter-laboratory coefficient of variation (CV) between 35 laboratories world-wide on 15 cerebrospinal fluid (CSF) samples. Critical factors, such as sample transport and storage, analytical delays, reaction temperature and time, the laboratories' accuracy and preparation of standards were documented and used for the statistical analyses.

Results: The intra-laboratory CV averaged 3.3\% and the interlaboratory CV 59\%. The results from the test laboratories correlated with those from the reference laboratory $(R=0.60, p<0.0001)$. Correcting for critical factors improved the strength of the correlation. Differences in the accuracy of standard preparation was identified as the most critical factor. Correcting for the error introduced by variation in the protein standards improved the correlation to $\mathrm{R}=0.98, \mathrm{p}<0.0001$ with an averaged inter-laboratory CV of $14 \%$. The corrected overall inter-rater agreement was subtantial (0.6) according to Fleiss' multirater kappa and Gwet's AC1 statistics.

Conclusion: This multi-center validation study identified the lack of preparation of accurate and consistent protein standards as the main reason for a poor inter-laboratory CV. This issue is also relevant to other protein biomarkers based on this type of assay and will need to be solved in order to achieve an acceptable level of analytical accuracy. The raw data of this study is available online.
\end{abstract}

keywords: neurofilament, NfL, NF-L, NEFL, biomarker, validation 


\section{Introduction}

Protein biomarkers are relevant for diagnostics and prognosis and they are increasingly used as inclusion criteria and as a secondary outcome measure for treatment trials. With the availability of high-throughput proteomic methods large numbers of potential protein biomarkers have been discovered. However, surprisingly few withstand the test of time and bridge the bench to bedside gap. Two examples within the field of neurodegenerative diseases are the tau and amyloid-beta ${ }_{1-42}$ proteins. Both proteins were found to be key components in the pathology of Alzheimer's disease. Several assays were developed, of which the enzyme-linked immunoabsorbant assays (ELISA) were employed routinely. Evidence emerged that cerebrospinal fluid (CSF) tau and amyloid-beta ${ }_{1-42}$ levels could be good diagnostic tests for Alzheimer's disease [1,2] and new diagnostic criteria including CSF tau and amyloid-beta ${ }_{1-42}$ levels were proposed [3]. Disappointingly, repeated validation studies of CSF tau and amyloid-beta ${ }_{1-42}$ levels demonstrated an unacceptable inter-laboratory coefficient of variation $(\mathrm{CV})$ of up to $53 \%[4,5,6]$. The factors influencing the $\mathrm{CV}$ remain largely unknown.

The present study was designed to measure the quantitative influence of a number of controllable parameters in a multicenter study involving 35 laboratories worldwide. The rationale was to control for parameters inherent to shipment and handling of test material and related to different laboratory procedures in order to optimize the inter-laboratory CV.

The biomarker chosen for analysis was the neurofilament light protein (NfL) [7, 8]. Neurofilaments are heteropolymers composed of at least four subunit proteins: $\alpha$-internexin/Nf66 and the triplet of the neurofilament light, medium (NfM) and heavy chain $(\mathrm{NfH})[9,10]$. Neurofilament proteins are the most reliable protein biomarker validated for quantification of neurodegeneration $[10,11]$. Their emerging importance for basic science, clinical trials and laboratory practice cannot be overestimated.

\section{Materials and methods}

\subsection{Kits and equipment}

The NF-L kits were obtained from UmanDiagnostics, Umea, Sweden. The kit is a sandwich ELISA with two highly specific NF-L monoclonal antibodies and no cross-reactivity with other known brain antigens [12, 13, 14]. 
Large thermometers, allowing easy visualisation of the temperature (Table 1), were purchased from the Lab Warehouse (TG835-15). All thermometers were calibrated in London prior to shipment.

\subsection{CSF samples}

Fifteen CSF samples (50 mL each sample, collected from patients who underwent extraventricular drainage for management of acute hydrocephalus) were collected in polypropylene tubes and immediately centrifuged (5000 rpm for 5 minutes). Supernatants were aliquotted and stored at $-80^{\circ} \mathrm{C}$ in 1.5-2.0 mL Eppendorf tubes until analysis. In agreement with the Ethic Committee and the United Kingdom Human Tissue Act, all patient details were anonymised.

\subsection{Analytical procedures}

The ELISA (UmanDiagnostics NF-light $\AA$ ) was performed as previously described [13]. In brief, each laboratory reconstituted each of the freezedried NfL standards (purified bovine spinal cord with a purity $>98 \%$ ) with $240 \mu \mathrm{L}$ of sample diluent in a $1.5 \mathrm{~mL}$ Eppendorf vial to give a concentration of $40 \mathrm{ng} / \mathrm{mL}^{79}$. The vial was gently mixed until the standard was completely solubilized and then left to stand at room temperature (RT) for 10 minutes. A series of eight Eppendorf vials was prepared, with the first vial containing $900 \mu \mathrm{L}$ of sample diluent and vials 2 to $8,600 \mu \mathrm{L}$ of sample diluent. Each laboratory then added $300 \mu \mathrm{L}$ of the lyophilized standard to the first vial to give a concentration of $10 \mu \mathrm{g} / \mathrm{L}$. A doubling dilution using $600 \mu \mathrm{L}$ in each step was performed and vial 8 was left only with sample diluent to give the blank reading $(0 \mathrm{ng} / \mathrm{mL} \mathrm{NfL})$. The concentration for vials 1 to 7 is summarised in Table 3.

The freeze-dried ELISA plates were washed with $3 \times 5$-minute cycles with washing solution (300 $\mu \mathrm{L}$ ). Next, $100 \mu \mathrm{L}$ of standards and samples were added in quadruplicate (= 4 measurements) and incubated for 1 hour at RT at $800 \mathrm{rpm}$ agitation on a shaker. After another three 5minute wash cycles $100 \mu \mathrm{L}$ of the biotin-labeled anti-NfL monoclonal antibody were added to each well. The plate was incubated for 45 minutes (RT, 800 rpm agitation). Following another wash cycle ( $3 \times 5$ minutes), 100 $\mu \mathrm{L}$ of streptavidin-HRP conjugate were added to each well. The plate was incubated for 30 minutes (RT, $800 \mathrm{rpm})$. A last wash cycle $(3 \times 5$ minutes)

\footnotetext{
${ }^{79}$ The volume of stock solution for the two freeze-dried NfL standards amounts to 480 $\mu \mathrm{L}$
} 
was performed and $100 \mu \mathrm{L}$ of 3,3',5,5'-tetramethylbenzidine (TMB) were added to each well. After incubating the plate for 15 minutes (RT) the color reaction was stopped with $50 \mu \mathrm{L}$ of stop solution $\left(0.18 \mathrm{M} \mathrm{H}_{2} \mathrm{SO}_{4}\right)$. The absorbance (optical density, OD) of each well was read at $450 \mathrm{~nm}$ (test) and $700 \mathrm{~nm}$ (blank).

All laboratories performed only one run.

\subsection{Data analysis and strategy for validation}

The raw data was sent to London (AP) for analysis and to Amsterdam (CT) for independent control. The concentration of the NfL samples was calculated based on each laboratory's standard curve using the closest three ODs of the quadruplicates. The results were then discussed with each laboratory. One dataset was again sent to Amsterdam (CT) for independent control. Results from samples which gave a higher OD than the highest standard were excluded as this study did not allow for extrapolation. All raw data were then subjected to statistical analysis.

SAS software (version 9.2, SAS Institute, Inc., Cary, North Carolina, USA) was used for all statistical analyses and graphs. Data was presented as median and interquartile range (IQR). The coefficient of variation (CV) was calculated for each measurement as the standard-deviation divided by the mean. The result was then multiplied by 100 for expression as a percentage. Correlation analyses were performed using Pearson's $R$ for normally distributed and Spearman's R for non-Gaussian data. Partial correlation analyses were performed on predefined variables: temperature at which the ELISA was performed, time of the colorimetric reaction, time delay between shipment and analysis of samples, accuracy of each laboratory in respect to preparation of standards and intra-assay CV. The data for each sample was ranked in order to analyse the inter-laboratory agreement. The inter-laboratory agreement was assessed using kappa statistics for multiple raters and multiple samples [15] and rated as described [16]. The Fleiss' multiple rater kappa was calculated on those samples successfully measured by each laboratory using the SAS magree macro (version 1.2). The conditional kappa for each response category as well as the overall agreement are presented. In addition, kappa coefficients were also calculated using the more robust $A C 1$ statistics introduced by Gwet [17] using the SAS inter_rater macro (version 1.0, downloaded from http://www.stataxis.com/files/sas/INTER_RATER.TXT). 


\section{Results}

The samples were shipped on dry ice and arrived in perfect frozen storage conditions in $34(97 \%)$ of the laboratories (Table 1). In one case the samples were delayed at an airport for 2 days and arrived defrosted (laboratory \#26). Samples were stored at $-80^{\circ} \mathrm{C}$ by 33 of the laboratories until analysis. The average delay from shipment of samples to analyses was two weeks (Table 1). All laboratories used yellow top (100-200 $\mu \mathrm{L}$ ) pipettes for pipetting samples and standards into the ELISA plate. These pipettes were specifically calibrated prior to the experiment by $51 \%$ of laboratories. In all other cases pipettes were calibrated on a routine basis as part of normal laboratory practice. For the preparation of standards $83 \%$ of laboratories used a different set of pipettes, which were able to handle larger volumes (up to $1000 \mu \mathrm{L}$ ).

The ELISA kit worked successfully in 33/35 (94\%) of the participating laboratories. In two laboratories, the ELISA did not work due to very low optical densities $(\mathrm{OD}<0.01)$ in one, probably reflecting a pipetting error due to mismatch of antibodies. Almost uniformly high ODs were found by another laboratory (\#14), probably reflecting cross-contamination. The temperature at which the ELISA was performed averaged $25^{\circ} \mathrm{C}$ (Table 1). The time given for the colorimetric reaction averaged 14 minutes (median 15 minutes).

In nine laboratories the standard curve went into saturation. In these cases the top standard and a samples with a high NfL concentration (samples \#1, \#5, \#6, \#9, \#10,\#13 and \#14, Figure 1) had to be excluded. In this study it was not allowed to extrapolate and therefore we were unable to calculate the concentration of a sample which fell outside the range of the standard curve. For this reason the NfL concentration could not be calculated from the optical density of $203 / 801(26.5 \%)$ of all samples (Table 2). The failure rate was highest for sample \#5 (33/2, 94.3\%). In contrast, the NfL concentration could be calculated from the results of all laboratories for sample \#4 (35/35, 100\%, Figure 1). Table 2 summarises the success rate for determining the $\mathrm{OD}$ for each sample and the failure rate for calculating the absolute concentration. The percentage agreement for the ranking of samples was higher if the ranking was based on OD instead of protein concentration for 6 samples (\#3, \#7, \#8, \#10, \#11, \#12). The agreement between laboratories for individual samples ranged from fair (sample \#2) to almost perfect (sample \#4, Table 2). The overall agreement was substantial [16] using either Fleiss' multi-rater kappa (overall kappa $0.602, p<0.0001$ ) or Gwet's AC1 statistics (overall conditional AC1 0.595, 
standard error 0.081, $\mathrm{p}<0.0001$; unconditional AC1 0.595, standard error $0.084, p<0.0001$.).

The overal ranking of the absolute protein concentration was identical to what was found for the OD (Table 2). But only for one sample (\#4) a result was obtained from all laboratories, preventing calculation of the inter-laboratory agreement using kappa statistics.

\subsection{Intra-laboratory assay CV}

The average intra-laboratory assay CV was 3.3\% (median $2.7 \%$ ). The breakdown for each standard and sample is summarised in Figure 2 . The intra-assay CV was best for high concentrations of NfL (standard 1: 10,000 $\mathrm{ng} / \mathrm{mL}$ ) and worst for low concentrations of $\mathrm{NfL}$ (standard 8: $0 \mathrm{ng} / \mathrm{mL}$ ).

\subsection{Inter-laboratory assay CV}

The concentration of NfL was calculated based on each laboratory's standard curve. Figure 1 shows that there was a large discrepancy in the reported values between the laboratories. This translated to an average inter-laboratory assay CV of 59\% (median 47\%, Figure 3).

\subsection{Validation parameters}

This validation study was designed to assess the influence of quantifiable parameters on the inter-laboratory assay CV. The values of all laboratories were compared to those from the reference laboratory (London). Using all CSF samples, there was a weak correlation of the absolute NfL concentration of $R=0.60(p<0.0001)$.

Temperature The partial correlation analysis demonstrated the influence of the temperature at which the assay was performed with $\mathrm{R}$ decreasing to $0.58(p<0.0001)$.

Time Also the time of color development had little influence on the results. Correcting for this variable in the partial correlation analysis, $R$ increased marginally to $0.62(p<0.0001)$.

Delay in analysis The time delay between sending out the samples and analysis had no effect on the results $(R=0.60, p<0.0001)$. 
Laboratory accuracy as estimated by the intra-assay CV The intraassay CV (accuracy of each laboratory) had no effect $(R=0.60, p<0.0001)$.

Preparation of standards The greatest influence on inter-laboratory validation was found to be variation in the concentration of the standards used by each laboratory to calculate the CSF NfL concentration of the samples. This is illustrated by two laboratories which analyzed the samples only two days apart at almost equal temperature $\left(26^{\circ} \mathrm{C}\right.$ and $27^{\circ} \mathrm{C}$, Table 3). The two standard curves are not parallel and contain different concentrations of NfL. Surprisingly the optical densities for the CSF samples are almost identical (0-2\% difference, Table 3). However, the reported concentration of CSF NfL differed considerably: $43-53 \%$ (Table 3).

It is possible that a non-systematic error was introduced because $84 \%$ of the laboratories used different pipettes to prepare the standards and pipette the samples (Table 1). In order to analyse the potential error introduced by differences in the concentration of the NfL standard, the CSF samples were normalized separately to the highest optical density for each laboratory (a one point callibration to a top value of 1). Comparing these normalised ODs, the correlation of the results improved to $R=0.72$ $(p<0.0001)$.

Figure 1 shows that not all laboratories measured the highest value for the same sample and the inter-laboratory rater agreement was not perfect for all samples (Table 2). In order to standardise we selected one sample in which the protein concentration was available from most of the participating laboratories. In addition, this sample had a sufficient high protein concentration to allow for normalisation of the remaining samples. We did therefore chose to normalise for sample 7 (82\% success rate for protein concentration, Table 2). Sample 7 had an average NfL concentration higher then in six other samples (\#2, \#3, \#4, \#8, \#11, \#12) with sufficient success rates to allow for a correlation analysis.Standardizing the normalization procedure to CSF sample 7 further improved the correlation to $R=0.98(p<0.0001$, Figure 4$)$.

\subsection{Optimised inter-laboratory assay CV}

Optimising the results to the largest effect found (standardisation to CSF sample 7), due to a non-systematic error in preparation of protein standards, reduced the inter-laboratory assay CV to an average of $14 \%$. Again, a better CV was found for CSF samples with a high NfL protein concentration (Figure 5). 


\section{Discussion}

This validation study identified parameters influencing the inter-laboratory CV. In descending importance these were the preparation of the standard curve, time of the colorimetric reaction, delay between shipment and sample analysis, intra-laboratory $\mathrm{CV}$, and temperature during the experiment. The importance of identifying these factors is highlighted by the strength of the correlation between the results from the test and reference laboratories. For uncontrolled conditions there was only a weak correlation with $\mathrm{R}=0.60$ with an inter-laboratory $\mathrm{CV}$ of $64.8 \%$. Under controlled conditions this was improved to a correlation of $\mathrm{R}=0.98$ with an inter-laboratory $\mathrm{CV}$ of $14 \%$. Under optimal conditions an inter-laboratory CV of less than $15 \%$ should be achievable.

The most important finding of this study is the influence of the preparation of the standards. Controlling for all other parameters, this can account for a two-fold difference in reported values. This variation is in part explained by limited accuracy of the pipettes. Only half of the laboratories calibrated their pipettes prior to the experiment. The other half calibrated their pipettes on a regular basis, which is generally regarded as good laboratory practice. A limitation of our study design is that this information was self-reported and could therefore be a source of unaccounted variation.

One peculiarity of NfL is that it can self-assemble [18, 19, 20,21]. Thus the formation of aggregates is a possibility. Aggregates can be dissolved either with time or using sonication. A difference in the solubilisation of the lyophilized NfL reference probably accounts for a large part of discrepancies between laboratories. We suspect that differences between laboratories in the preparation of the standards is also the likely cause of the unacceptable high inter-laboratory $\mathrm{CV}$ in other studies $[4,5,6]$. At present this key factor is not controlled for by any of the available ELISA kits for protein biomarkers. It is important to note that the inter-rater reliability is typically measured when all raters (e.g. laboratories) operate under similar conditions. Therefore the identification of a new extragenous factor has two implications. Firstly, the statistical strength of the kappa coefficient is limited. Secondly, a future controlled experiment should be conducted to evaluate the effect of standard preparation on the agreement coefficient.

All enzymes work within a range of temperature. The enzyme used in this experiment was horseradish peroxidase (HRP). The colorimetric reaction depends on the oxidation of 3,3',5,5'-tetramethylbenzidine (TMB) and is optimal around $25^{\circ} \mathrm{C}$ at $\mathrm{pH}$ 7.0. All experiments were performed under ideal thermal conditions and correcting for temperature did not improve the 
results.

The enzyme activity is calculated as moles of substrate converted per time unit. Because the time kinetics for HRP are not completely linear, variation in reaction time can influence the outcome of the experiment, particularly if the reaction reaches saturation. In this study saturation was reached for a number of samples. As our partial correlation analysis shows correcting for time did only have a very small effect on the data.

The delay between shipment of samples and analysis is important for protein biomarkers, which have a limited stability. NfL has been found to be stable at $-80^{\circ} \mathrm{C} 10$ years after sampling [22]. Consistent with this data a delay in time from shipment to analysis was not statistically relevant.

There are substantial limitations to this study. Firstly, it was not possible to control for all extragenous factors. We have summarised all extragenous factors collected in form of a table. Secondly, the effect of the now identified relevance of standard prepartion has not been tested prospectively in a controlled experiment. We intend to address this issue in a future study. Thirdly, a number of observations have been self-reported (e.g. callibaration of pipettes). Because the participating laboratories are located in different countries and the limited experimental time, it will remain challenging to collect this information by one single observer. Fourth, to keep this manuscript focused and transparent limited use of statistical analyses was considered necessary. The raw data of our study has therefore been made available online. We are keen to hear about alternative statistical approaches to this complex issue, particularly in dealing with missing ratings which will remain a problem for this type of reliability studies. Many of the present statistical analyses were correlative. It needs to be borne in mind that a statistical correlation does not proof causality.

This large validation study on an ELISA for a protein biomarker demonstrated that the experience and accuracy (intra-assay CV) of each laboratory had only a minor influence on the outcome. This is reassuring, meaning that any laboratory should be in the position to use the ELISA, but will need to establish in-house reference ranges. In a post-hoc analysis the correlation analysis on the absolute concentration of CSF NfL levels between two laboratories experienced in the development of this type of ELISA (London and Götheborg), yielded an R of $0.99(p<0.0001)$. Our conclusion from this result is that at present any treatment trial including $\mathrm{NfL}$ as a protein biomarker may benefit from batch analyses in one laboratory to ensure the highest degree of accuracy. The low intra-laboratory $\mathrm{CV}$ is encouraging in this respect. Alternatively, the use of an internal laboratory quality control (such as sample 7 in this study), is strongly recommended. This study is consistent with previous data showing that hu- 
man factors are an important factor adding to inaccuracy [23]. Our results therefore highlight the importance of a careful adherence to the analytical procedure. The routine application of this ELISA will require carefull training of staff including calibration procedures in order to minimise the intra-laboratory inter-assay CV.

In summary this validation study on an ELISA for the quantification of NfL from human CSF revealed difficulties, due to the preparation of accurate and consistent standards. Even though an unacceptable poor inter-laboratory assay CV is known also for other proteins, such as tau and amyloid-beta ${ }_{1-42}[4,5,6]$, this is not yet controlled for. Under optimal conditions an inter-laboratory CV of $14 \%$ should be achievable for this NfL ELISA.

\section{Acknowledgments}

We would like to acknowledge the technical assistence of Mrs Ute Schulz, Mrs Brigitte Fritz, Mr Willy Deleersnijder, Ms Carmine Tamborino, MS Päivi Räsänen, Mrs W. van Geel, Ms Anh Dang, Ms Miliam Karamaga, Ms Martina Hoelzl, Ms Manjit Braitch, Mrs Olga Petropoulou, Ms Keresztes and Ms Lajtos. We would like to thank Dr Geoffrey Keir for discussion in the planing phase of the study and Prof Antonio Bertolotto for helpful comments on the manuscript.

This study received no financial support. All NF-light ${ }^{\circledR}$ were donated by UmanDiagnostics. Samples, packing and logistics were provided by the London laboratory and costs for shipment were paid by the participating laboratories.

\section{References}

[1] K. Blennow, M.J. de Leon, and H. Zetterberg. Alzheimer's disease. Lancet, 368:387-403, 2006.

[2] P. Lewczuk and J. Wiltfang. Neurochemical dementia diagnostics: State of the art and research perspectives. Proteomics, 8:1292-1301, 2008.

[3] B. Dubois, H. H. Feldman, C. Jacova, S. T. Dekosky, P. BarbergerGateau, J. Cummings, A. Delacourte, D. Galasko, S. Gauthier, G. Jicha, K. Meguro, J. O'brien, F. Pasquier, P. Robert, M. Rossor, 
S. Salloway, Y. Stern, P. J. Visser, and P. Scheltens. Research criteria for the diagnosis of Alzheimer's disease: revising the NINCDSADRDA criteria. Lancet Neurol, 6(8):734-46, 2007.

[4] MA Blankenstein, C Clark, De Deyn PP, Galasko D, Hartmann T, Hampel H, Kapaki E, Mehta PD, Parnetti L, Pirttila T, Verbeek MM, Wildfang J, Blennow K, and Verbeek MM. Multicenter comparison of assays for cerebrospinal fluid (CSF) markers for Alzheimer disease. In Clin Chim Acta, volume 355, page S175, 2005.

[5] P. Lewczuk, G. Beck, O. Ganslandt, H. Esselmann, F. Deisenhammer, A. Regeniter, H.F. Petereit, H. Tumani, A. Gerritzen, P. Oschmann, J. Schroder, P. Schonknecht, K. Zimmermann, H. Hampel, K. Burger, M. Otto, S. Haustein, K. Herzog, R. Dannenberg, U. Wurster, M. Bibl, J.M. Maler, U. Reubach, J. Kornhuber, and J. Wiltfang. International quality control survey of neurochemical dementia diagnostics. Neurosci Lett, 409:1-4, 2006.

[6] NA Verwey, WM van der Flier, K Blennow, C Clark, S Sokolow, PP De Deyn, D Galasko, H Hampel, T Hartmann, E Kapaki, L Lannfelt, PD Mehta, L Parnetti, A Petzold, T Pirttila, L Saleh, A Skinningsrud, JC v. Swieten, MM Verbeek, J Wiltfang, S Younkin, P Scheltens, and MA Blankenstein. A worldwide multicenter comparison of assays for CSF biomarkers in Alzheimer disease. Ann Clin Biochem. (in press).

[7] LE Rosengren, JE Karlsson, JO Karlsson, LI Persson, and C Wikkelso. Patients with amyotrophic lateral sclerosis and other neurodegenerative diseases have increased levels of neurofilament protein in CSF. J Neurochem, 67:2013-2018, 1996.

[8] WJA Van Geel, LE Rosengren, and MM Verbeek. An enzyme immunoassay to quantify neurofilament light chain in cerebrospinal fluid. J Immunol Meth, 296:179-185, 2005.

[9] G Shaw. Neurofilaments. Springer-Verlag, 1998.

[10] A Petzold. Neurofilament phosphoforms: surrogate markers for axonal injury, degeneration \& loss. J Neurol Sci, 233:183-198, 2005.

[11] C.E. Teunissen, C. Dijkstra, and C. Polman. Biological markers in CSF and blood for axonal degeneration in multiple sclerosis. Lancet Neurol, 4:32-41, 2005. 
[12] N. Norgren, J.E. Karlsson, L. Rosengren, and T. Stigbrand. Monoclonal antibodies selective for low molecular weight neurofilaments. Hybrid Hybridomics, 21:53-9, 2002.

[13] N Norgren, L Rosengren, and T Stigbrand. Elevated neurofilament levels in neurological diseases. Brain Res, 987(1):25-31, October 2003.

[14] N. Norgren, P. Sundstrom, A. Svenningsson, L. Rosengren, T. Stigbrand, and M. Gunnarsson. Neurofilament and glial fibrillary acidic protein in multiple sclerosis. Neurology, 63:1586-1590, 2004.

[15] JL Fleiss. Measuring nominal scale agreement among many raters. Psych Bull, 76:378-382, 1971.

[16] JR Landis and GG Koch. The measurement of observer agreement for categorical data. Biometrics, 33:159-174, 1977.

[17] A Gwet. Handbook of Inter-Rater Reliability. STATAXIS Publishing Company, Montgomery Village, MD 20886. USA, 2002.

[18] RK Liem and SB Hutchison. Purification of individual components of the neurofilament triplet: filament assembly from the 70 000-dalton subunit. Biochemistry, 21(13):3221-3206, June 1982.

[19] MK Lee, Z Xu, PC Wong, and DW Cleveland. Neurofilaments are obligate heteropolymers in vivo. J Cell Biol, 122(6):1337-1350, September 1993.

[20] J Carter, A Gragerov, K Konvicka, et al. Neurofilament (NF) assembly; divergent characteristics of human and rodent NF-L subunits. J Biol Chem, 273:5101-5108, 1998.

[21] R Perez-Olle, CL Leung, and RK Liem. Effects of Charcot-MarieTooth-linked mutations of the neurofilament light subunit on intermediate filament formation. J Cell Sci, 115:4937-4946, 2002.

[22] R. Constantinescu, M. Romer, D. Oakes, L. Rosengren, and K. Kieburtz. Levels of the light subunit of neurofilament triplet protein in cerebrospinal fluid in Huntington's disease. Parkinsonism Relat Disord, 2008.

[23] P. Carraro and M. Plebani. Errors in a stat laboratory: types and frequencies 10 years later. Clin Chem, 53:1338-1342, 2007. 
Table 1: Factors recorded for assay validation. The median (IQR), number and percentage are shown.

\begin{tabular}{ll}
\hline Factor & Description \\
\hline Number of participants & $n=35$ \\
Sample condition on arrival $\left({ }^{\circ} \mathrm{C}\right)$ & $-80(\mathrm{n}=34), 20(\mathrm{n}=1)$ \\
Sample storage prior to analyses $\left({ }^{\circ} \mathrm{C}\right)$ & $-80(\mathrm{n}=33),-40(\mathrm{n}=1),-20(\mathrm{n}=1)$ \\
ELISA kit storage prior to analyses $\left({ }^{\circ} \mathrm{C}\right)$ & $4(\mathrm{n}=33), 20(\mathrm{n}=2)$ \\
Delay to start of analysis (days) & $14(8-30)$ \\
Pipettes calibrated prior to experiment & $(18) 51 \%$ \\
$100-200 \mu$ L (yellow top) pipettes used for samples & $100 \%$ \\
Different pipettes used for preparing standards & $84 \%$ \\
Temperature at which ELISA was performed & $25.3(24.0-26.5)$ \\
Time of colour reaction (minutes) & $15(12-15)$ \\
\hline
\end{tabular}


Table 2: Success rates for measuring the $O D$ and calculating the absolute NfL protein concentration for each sample. The overall rank ( $1=$ lowest rank, $9=$ highest rank) of a sample is shown alongside the percentage of laboratories who ranked the sample equally (\% of labs). One laboratory (\#14) measured only one sample and was therefore not included into this analysis. The ranking was not calculated for ODs which were not measured by all of the 34 remaining laboratories. Fleiss' kappa and Gwet's AC1 statistics were calculated on the ranked OD. The conditional kappa for each response category is shown as well as the overall agreement (there was no difference between Gwet's conditional and unconditional AC1 statistics for this data, therefore only one column is presented.). Fleiss' kappa and Gwet's AC1 could not be calculated on the protein concentration due to a less then $100 \%$ success rate in 8 of 9 ranked samples. Not available $=-$

\begin{tabular}{|c|c|c|c|c|c|c|c|c|}
\hline \multirow{2}{*}{$\begin{array}{l}\text { Sample } \\
\text { number }\end{array}$} & \multicolumn{5}{|c|}{ OD } & \multicolumn{3}{|c|}{$\mathrm{NfL}[\mathrm{ng} / \mathrm{mL}]$} \\
\hline & Success & Rank & $\%$ of labs & Kappa & $\mathrm{AC} 1$ & Success & Rank & $\%$ of labs \\
\hline \#1 & $34 / 34(100 \%)$ & 9 & $72 \%$ & 0.47 & 0.46 & 23/34 (68\%) & 9 & $77 \%$ \\
\hline \#2 & $34 / 34(100 \%)$ & 6 & $65 \%$ & 0.38 & 0.36 & $29 / 34(85 \%)$ & 6 & $65 \%$ \\
\hline \#3 & $34 / 34(100 \%)$ & 3 & $68 \%$ & 0.47 & 0.45 & $33 / 34(97 \%)$ & 3 & $63 \%$ \\
\hline$\# 4$ & $34 / 34(100 \%)$ & 1 & $100 \%$ & 1.00 & 1.00 & $34 / 34(100 \%)$ & 1 & $100 \%$ \\
\hline \#5 & 26/34 (77\%) & - & - & - & - & 2/34 (6\%) & - & - \\
\hline \#6 & 32/34 (94\%) & - & - & - & - & 14/34 (41\%) & - & - \\
\hline \#7 & $34 / 34(100 \%)$ & 7 & $87 \%$ & 0.79 & 0.79 & 28/34 (82\%) & 7 & $77 \%$ \\
\hline \#8 & $34 / 34(100 \%)$ & 4 & $94 \%$ & 0.87 & 0.87 & $30 / 34(88 \%)$ & 4 & $89 \%$ \\
\hline$\# 9$ & 26/34 (77\%) & 一 & - & - & - & $5 / 34(15 \%)$ & - & - \\
\hline$\# 10$ & $34 / 34(100 \%)$ & 8 & $74 \%$ & 0.53 & 0.52 & $25 / 34(74 \%)$ & 8 & $67 \%$ \\
\hline$\# 11$ & $34 / 34(100 \%)$ & 5 & $62 \%$ & 0.42 & 0.41 & $29 / 34(85 \%)$ & 5 & $56 \%$ \\
\hline \#12 & $34 / 34(100 \%)$ & 2 & $68 \%$ & 0.47 & 0.46 & $32 / 34(94 \%)$ & 2 & $66 \%$ \\
\hline \#13 & 29/34 (85\%) & 一 & - & - & - & $7 / 34$ (21\%) & - & - \\
\hline \#14 & $31 / 34(91 \%)$ & - & - & - & - & 11/34 (32\%) & - & - \\
\hline \#15 & $33 / 34(97 \%)$ & 一 & 一 & 一 & - & $25 / 34(74 \%)$ & 一 & 一 \\
\hline Overall & - & - & - & 0.60 & 0.60 & - & - & - \\
\hline
\end{tabular}


Table 3: Preparation of standards was identified as the most important parameter influencing the inter-laboratory $\mathrm{CV}$. In this example the raw data from two laboratories closely matched for other parameters (temperature $27{ }^{\circ} \mathrm{C}$ and $26^{\circ} \mathrm{C}$, days between shipment and analysis 30 and 28 days, time of color reaction 15 and 8 minutes, respectively) are shown. Surprisingly, the optical densities for the CSF samples from these two laboratories are almost identical ( $0-2 \%$ difference). For the standards the $O D$ varies over a wider range (4-35\% difference). This is the reason for the large difference in the reported results of CSF NfL concentrations (43 to 53\%). The concentration for the NfL standards was defined as described in the analytical procedure. Note that for laboratory \#26 the mean OD of sample 1 (2.94) was higher than the mean OD for the highest standard (2.79). Because we did not allow for extrapolation in this study this sample would not have been included in the analyses.

\begin{tabular}{|c|c|c|c|c|c|c|}
\hline \multirow[t]{2}{*}{ Specimen } & \multicolumn{2}{|c|}{ Mean OD } & \multirow{2}{*}{$\begin{array}{l}\text { Difference } \\
\text { OD (\%) }\end{array}$} & \multicolumn{2}{|c|}{$\mathrm{NfL}(\mathrm{ng} / \mathrm{mL})$} & \multirow{2}{*}{$\begin{array}{l}\text { Difference } \\
\text { NfL (\%) }\end{array}$} \\
\hline & Lab \#26 & Lab \#18 & & Lab \#26 & Lab \#18 & \\
\hline Standard 1 & 2.79 & 3.12 & $11 \%$ & 10 & 10 & - \\
\hline Standard 2 & 1.89 & 2.92 & $35 \%$ & 5 & 5 & 一 \\
\hline Standard 3 & 1.22 & 1.72 & $29 \%$ & 2.5 & 2.5 & - \\
\hline Standard 4 & 0.66 & 0.92 & $18 \%$ & 1.25 & 1.25 & 一 \\
\hline Standard 5 & 0.40 & 0.47 & $15 \%$ & 0.625 & 0.625 & - \\
\hline Standard 6 & 0.23 & 0.22 & $4 \%$ & 0.313 & 0.313 & 一 \\
\hline Standard 7 & 0.14 & 0.11 & $11 \%$ & 0.156 & 0.156 & - \\
\hline Sample 1 & 2.94 & 2.96 & $1 \%$ & 10.899 & 5.075 & $53 \%$ \\
\hline Sample 7 & 2.35 & 2.31 & $2 \%$ & 7.394 & 3.675 & $51 \%$ \\
\hline Sample 11 & 1.74 & 1.74 & $0 \%$ & 4.484 & 2.574 & $43 \%$ \\
\hline
\end{tabular}




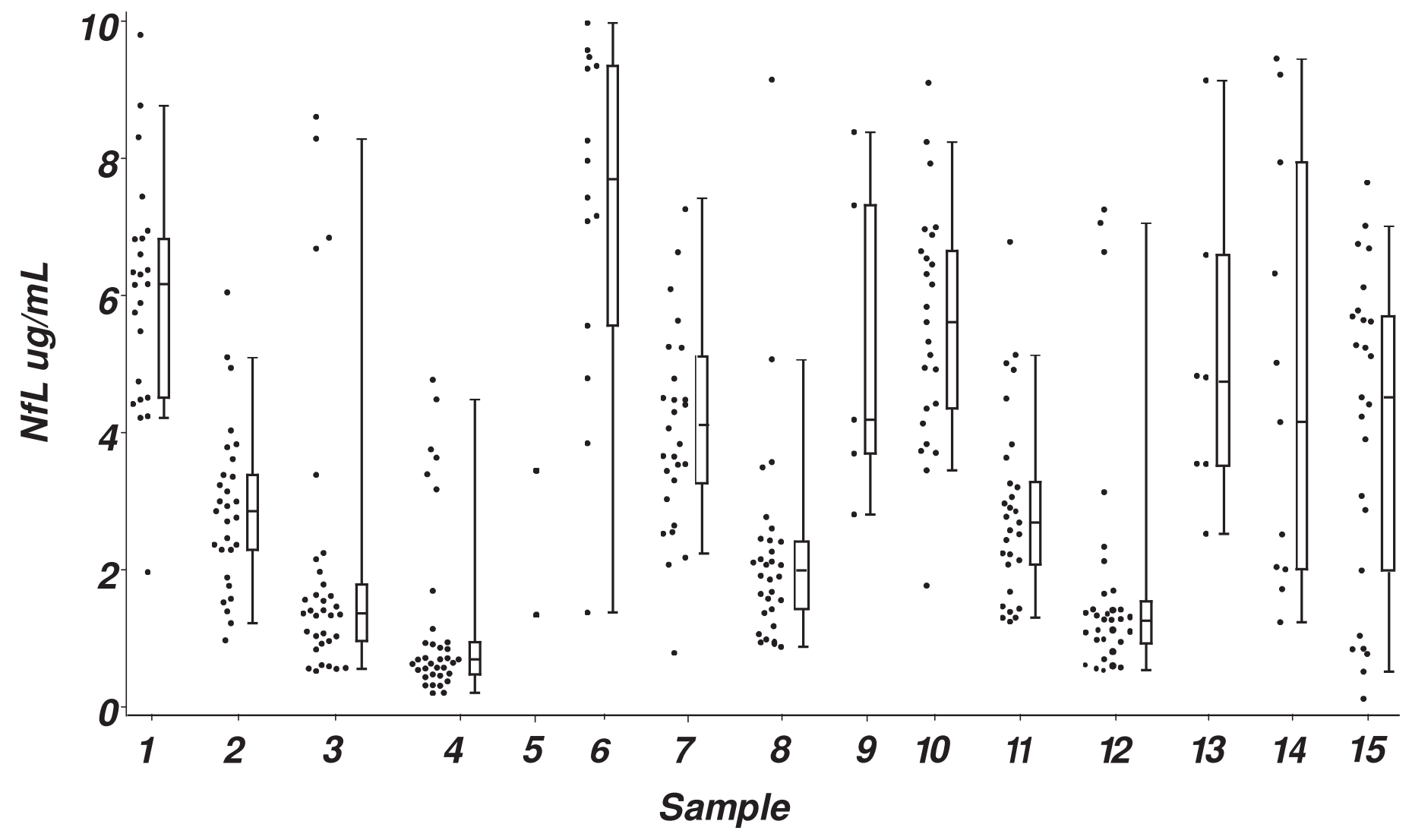

Figure 1: The concentration of CSF NfL levels $(\mu \mathrm{g} / \mathrm{mL})$ measured per sample is shown. Individual data points are represented by dots, the box-andwhisker boxes indicate the median and interquartile (25-75\%) range. 


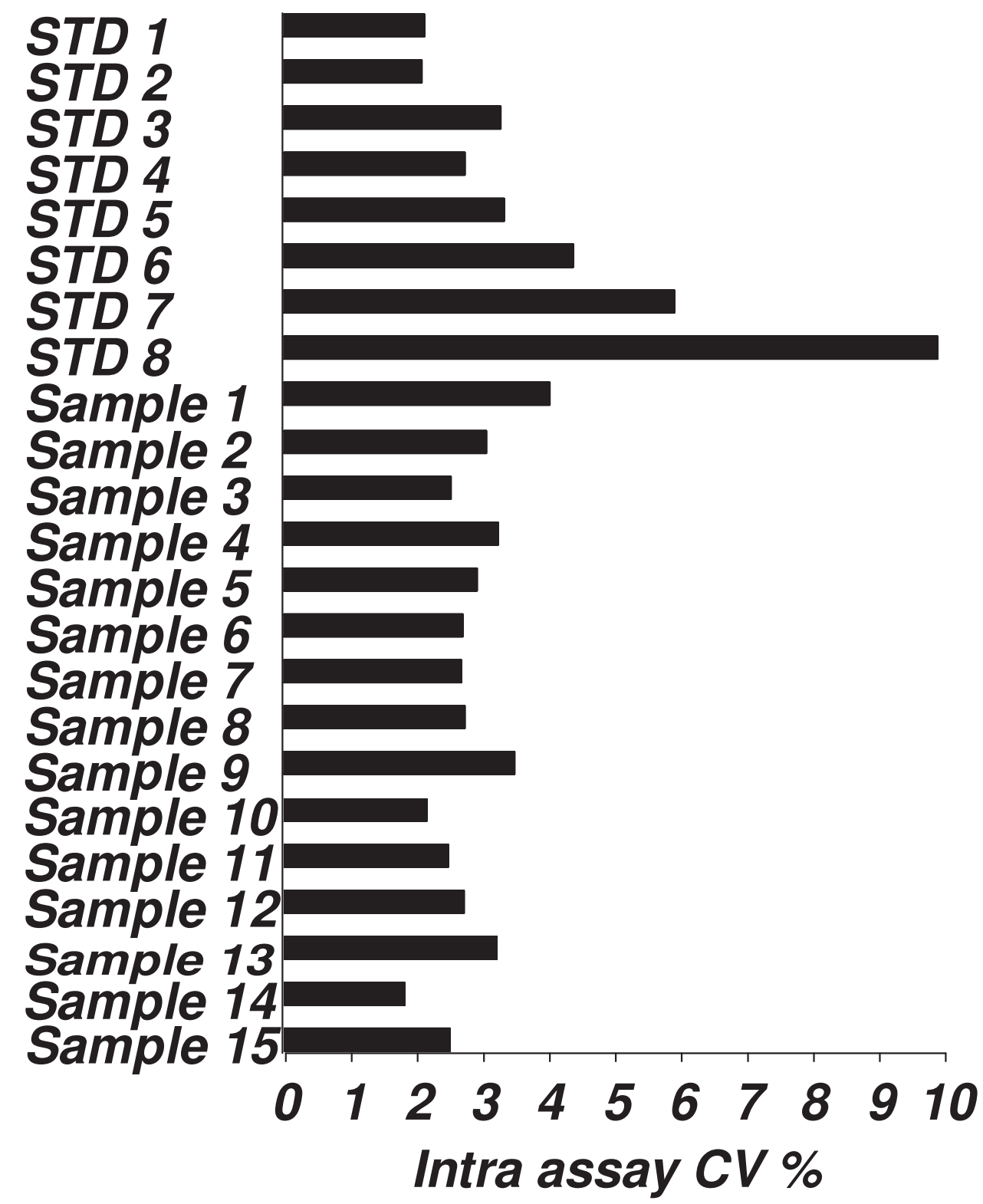

Figure 2: Intra-assay CV. The bar-chart shows the intra-assay CV for all standards and samples analyzed. The intra-assay CV averages to $4.0 \%$ (small horizontal bars). 


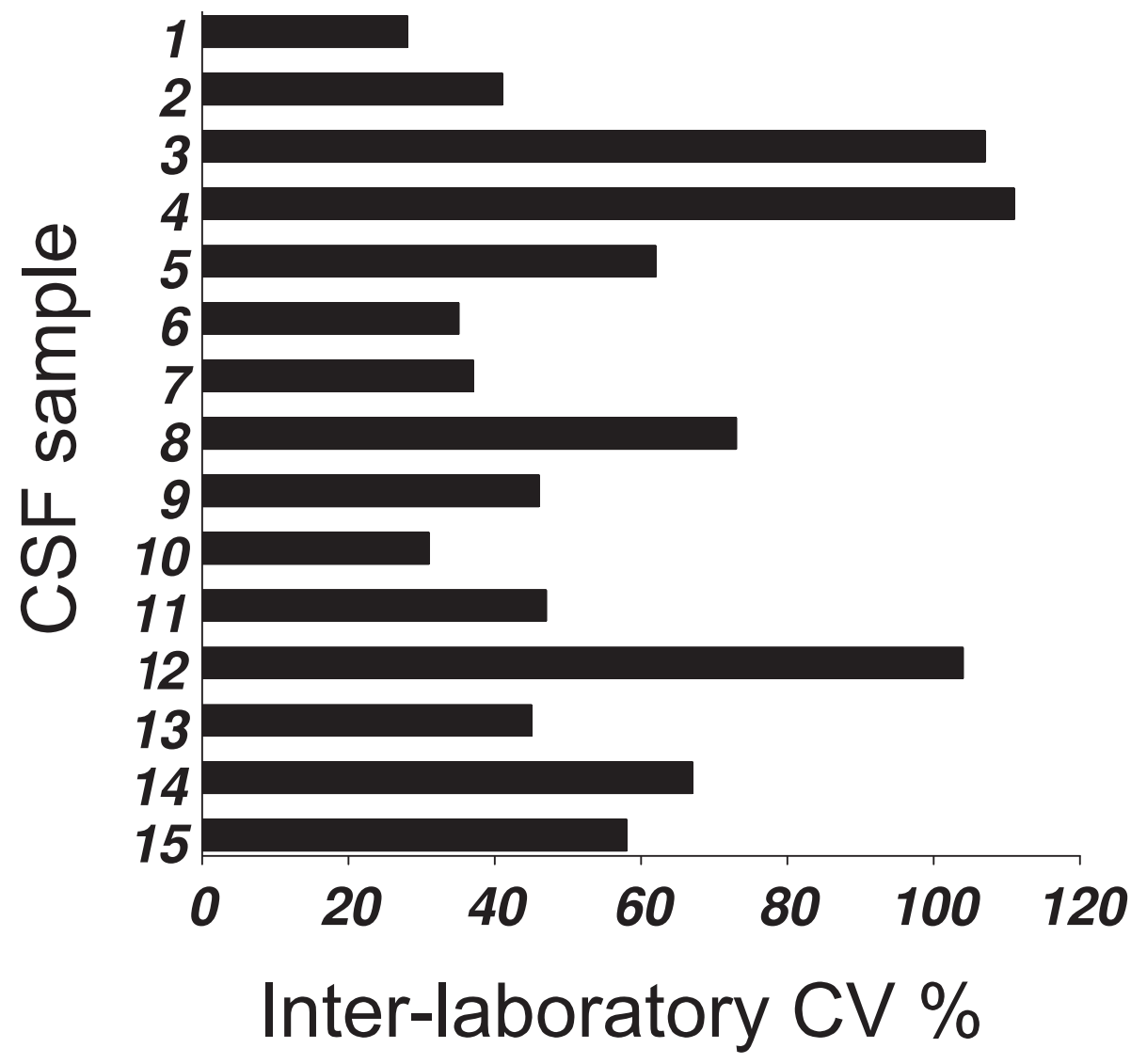

Figure 3: Inter-laboratory assay CV. The bar-chart shows the interlaboratory assay CV for each individual sample. The averaged interlaboratory assay $\mathrm{CV}$ is $64.8 \%$. 


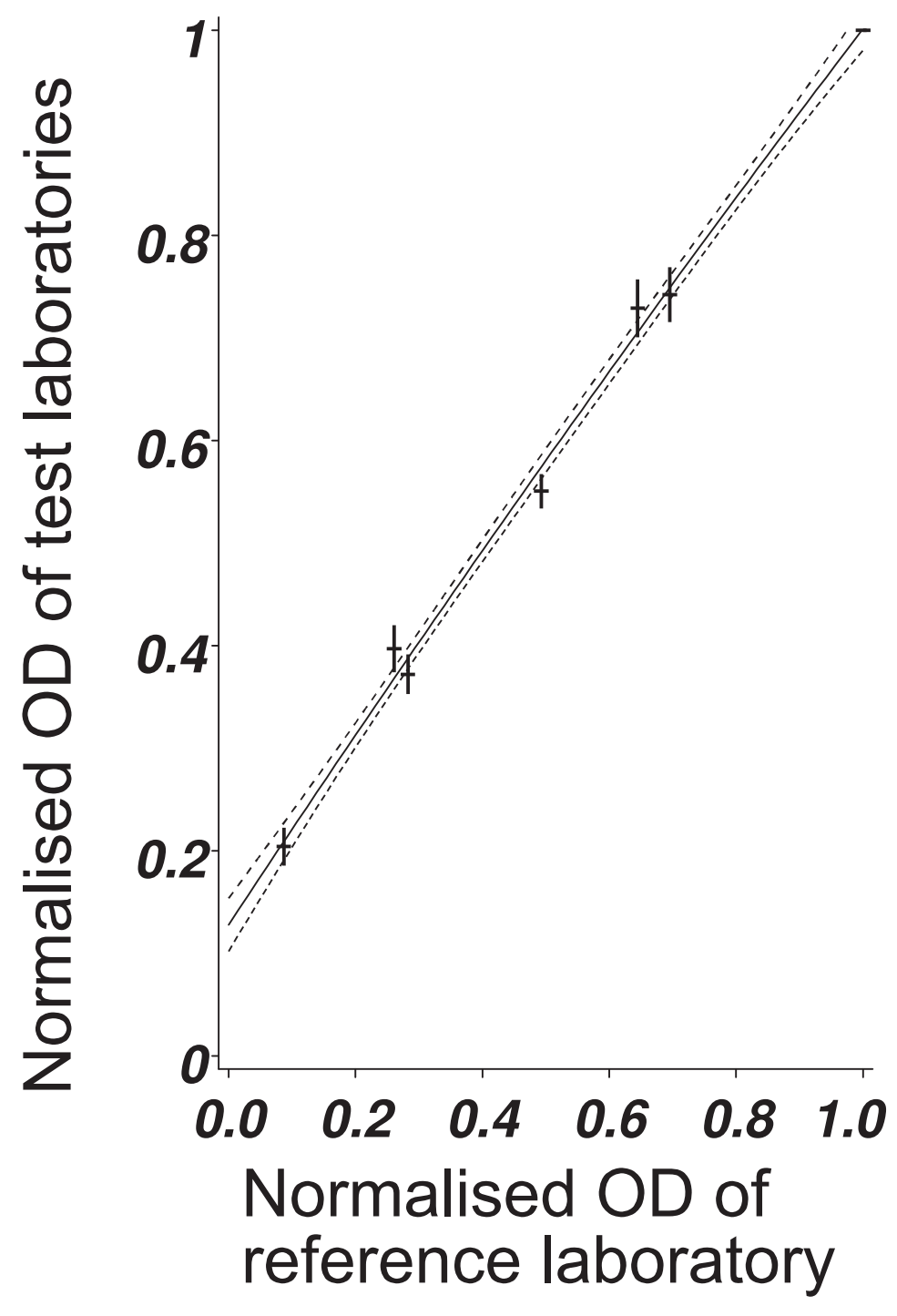

Figure 4: Correlation analysis on standardized normalization of the optical density to CSF sample \#7 $(R=0.98, p<0.0001)$. 


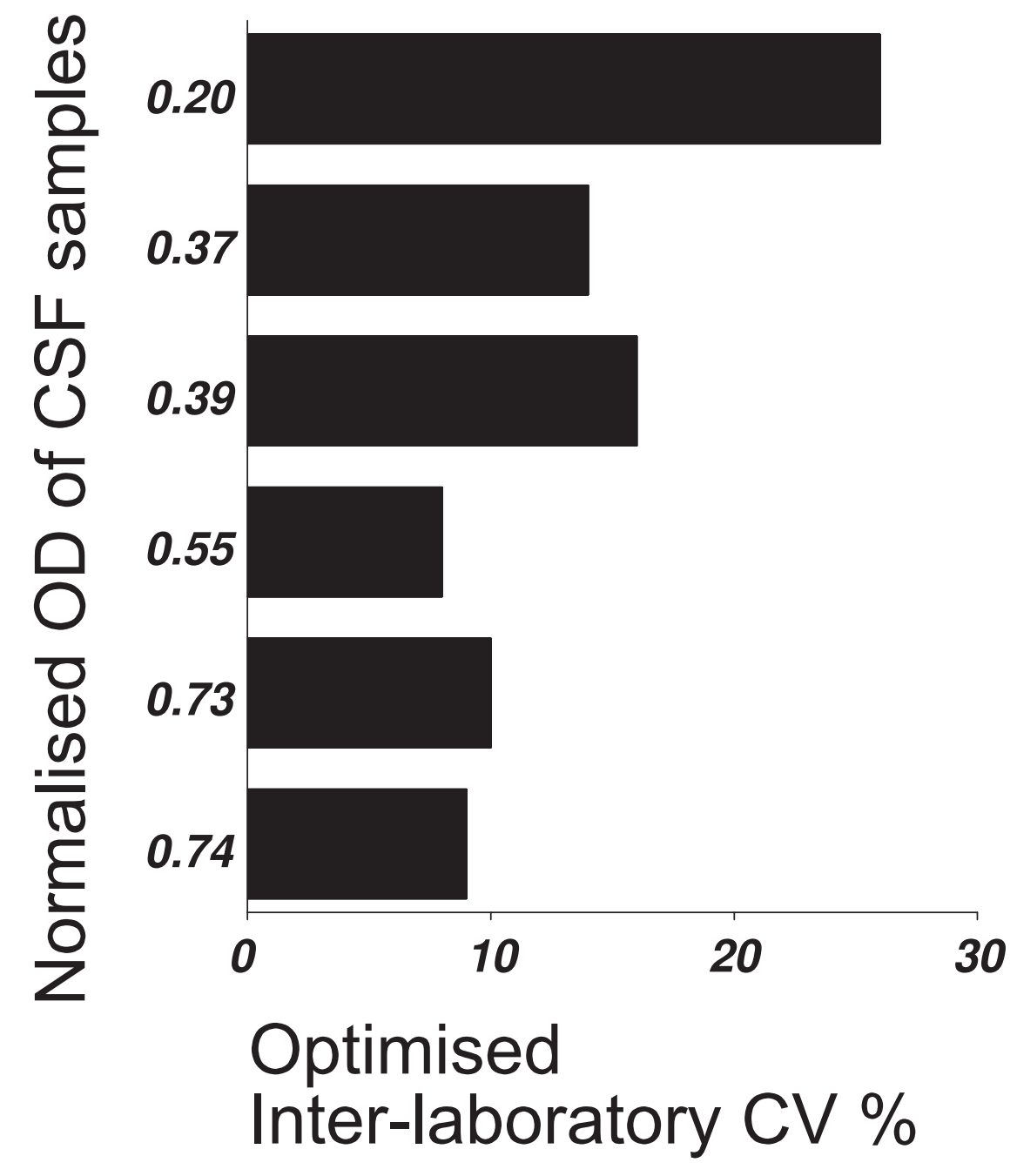

Figure 5: The optimized inter-laboratory CV, based on the normalized mean OD of the CSF samples as a series is shown. All CSF samples (\#2, \#3, \#4, \#8, \#11, \#12) were normalized to CSF sample \#7. The optimized inter-laboratory CV averages $13.8 \%$ (median 12\%). 